\title{
Aplicação de Ferramentas da Qualidade em um desvio de qualidade na indústria farmacêutica: um estudo de caso
}

\author{
Application of Quality Tools to solve a quality deviation in the \\ pharmaceutical industry: a case study
}

Recebido em: 15/06/2021

Aceito em: $19 / 10 / 2021$
Thais Santos AMORIM; Luís Henrique GARCIA-AMOEDO

Universidade Cruzeiro do Sul. Avenida Regente Feijó, 1295. Vila Regente Feijó, CEP 03342-000. São Paulo, SP, Brasil.

E-mail: thaisjks@gmail.com

\section{ABSTRACT}

Currently, due to the great competition among pharmaceutical companies, the demand for more quality, safety and efficient products has been increasing, generating the need for processes that are more robust and a qualified team. However, quality deviations can occur during the production process and the cause of this event must be identified and solved in order to avoid repeating mistakes. The objective of this study is to demonstrate through a case study the practical use of quality tools to identify and solve a quality deviation of broken pills in a pharmaceutical industry. For the organization, data evaluation and the identification of the root cause of this deviation, the quality tools of Pareto Diagram, Control Chart, Check List Sheet and Ishikawa Diagram were used. After data evaluation, it was possible to conclude that the breakage of the tablets is related to the low result of the hardness test, and then, it was suggested to the company to manufacture the next batches seeking hardness close to or greater than $200 \mathrm{~N}$, according to the product's history. Therefore, the quality tools proved to be essential for an effective assessment of the quality deviation.

Keywords: broken tablets; hardness test; pharmaceutical industry; quality

\section{RESUMO}

Atualmente, devido à grande concorrência entre as indústrias, houve um aumento de demanda por produtos mais eficientes, seguros e com excelência em qualidade, gerando a necessidade de processos mais robustos e equipe qualificada e capacitada. Entretanto, desvios de qualidade podem ocorrer durante o processo produtivo, e as causas destes eventos devem ser identificadas e solucionadas, a fim de evitar repetições dos erros. O objetivo deste trabalho foi demonstrar por meio de um estudo de caso, a utilização prática das ferramentas da qualidade para identificar e solucionar um desvio de qualidade de comprimidos quebrados em uma indústria farmacêutica. Para identificação da causa raiz do desvio, organização e interpretação dos dados, foram utilizadas ferramentas da qualidade, tais como Diagrama de Pareto, Gráfico de Controle, Folha de Verificação e Diagrama de Ishikawa. Após a avaliação dos dados por meio destas ferramentas, foi 
possível concluir que a quebra dos comprimidos está relacionada com o resultado baixo do teste de dureza, e, portanto, foi sugerido à empresa fabricar os próximos lotes buscando a dureza próxima ou superior a 200 $\mathrm{N}$, conforme histórico do produto. Sendo assim, as ferramentas da qualidade se mostraram essenciais para uma avaliação efetiva do desvio de qualidade.

Palavras-chave: comprimido quebrado; fabricação; teste de dureza; indústria farmacêutica; qualidade

\section{INTRODUÇÃO}

Devido à grande concorrência ocasionada pela globalização, as empresas vêm necessitando cada vez mais de processos robustos, de produtos seguros e com excelência em qualidade que levem uma visão positiva da marca em seus clientes e consumidores (1).

A Indústria Farmacêutica integra o setor responsável pela produção de medicamentos, e possui diversas áreas trabalhando em conjunto para atingir a excelência dos processos produtivos seguindo as determinações legais às quais a empresa deve acompanhar. Uma das áreas que garante o cumprimento das determinações legais é a Garantia de Qualidade, sendo este setor responsável por documentar, monitorar e auxiliar as atividades produtivas e de suporte da fábrica. Dentre as atividades para garantir a qualidade dos produtos, existe o comprometimento para com a investigação de desvios de qualidade (2).

Conforme a Resolução da Diretoria Colegiada (RDC) da Agência Nacional de Vigilância Sanitária (Anvisa) no 301, de 21 de agosto de 2019, desvio de qualidade é um não cumprimento dos requisitos estabelecidos pelo sistema de gestão da qualidade ou necessários para a manutenção da qualidade, segurança e eficácia dos produtos, ou seja, são possíveis ocorrências durante a fabricação, transporte ou armazenamento dos produtos podendo afetar os parâmetros de qualidade estabelecidos (3).

Mesmo com uma equipe capacitada e controles durante os processos, desvios ainda assim podem ocorrer, e os responsáveis pela sua investigação devem ser pessoas treinadas e capacitadas $(4,5)$.

O sistema de gerenciamento de desvios deve ser capaz de assegurar que todas as não conformidades sejam identificadas, reportadas e registradas, para então serem avaliadas quanto à sua criti- cidade, causa raiz e ações corretivas e preventivas (3). Dessa forma, evita-se a recorrência da falha, e consequentemente o desperdício de mão de obra e matérias primas que causam impacto no lucro da empresa (1).

Existem diversas ferramentas da qualidade que podem ser utilizadas durante a investigação do desvio de qualidade, e que auxiliam na análise e monitoramento das falhas investigadas. Dentre essas ferramentas, pode-se citar as ferramentas básicas da qualidade, como a folha de verificação, diagrama de Ishikawa, diagrama de Pareto e carta de controle (2).

Este estudo de caso tem como objetivo abordar e demonstrar a aplicação das ferramentas da qualidade quando da investigação de um desvio de qualidade relacionado à quebra de comprimidos durante a fabricação de um lote. $\mathrm{O}$ cenário se desenvolveu em uma indústria farmacêutica de grande porte localizada na região metropolitana de São Paulo, que possui um sistema consolidado de gestão e gerenciamento de desvios de qualidade, cuja investigação ocorre de forma dinâmica entre a garantia da qualidade e o setor em que o desvio ocorreu.

\section{MÉTODO}

Este estudo de caso apresenta um exemplo de utilização das ferramentas de qualidade em uma indústria farmacêutica multinacional localizada na região metropolitana de São Paulo, que produz medicamentos, cosméticos e suplementos alimentares na unidade fabril em que o estudo foi conduzido.

Após a identificação do desvio de qualidade relacionado a comprimidos quebrados, foi realizado um estudo exploratório misto para identificação e solução do problema por meio de ferramentas da qualidade, utilizando de entrevista aos funcio- 
nários, além de consulta de dados de produção, artigos científicos e livros. A indústria em questão disponibilizou todos os dados quantitativos e qualitativos solicitados acerca do desvio de qualidade para serem analisados através das ferramentas da qualidade.

Para análise e solução dos problemas, foram utilizadas as ferramentas da qualidade como folha de verificação, diagrama de Ishikawa, diagrama de Pareto e carta de controle. Como ferramenta para organização dos dados foi utilizado o programa Microsoft Office Excel 2016.

Devido à confidencialidade, o produto que apresentou o desvio foi nomeado como " $\mathrm{X}$ " e a descrição dos lotes está identificada como ano/ produto/lote no ano, como por exemplo, o primeiro lote de 2019 está descrito como 2019X01.

\section{RESULTADOS E DISCUSSÃO}

Após a finalização da produção do medicamento X (lote 2020X01) cuja forma farmacêutica é comprimido sem revestimento, foi realizada amostragem representativa conforme a validação vigente para o produto a fim de verificar possíveis defeitos como comprimidos manchados, quebrados, entre outros.

Conforme a RDC n ${ }^{0} 301$, de 21 de agosto de 2019, por meio da validação, pode-se provar que todo processo que utiliza materiais e equipamentos definidos origina o produto conforme a qualidade exigida e esperada (3).

Durante a avaliação da amostragem, foram identificados 35 comprimidos quebrados em uma amostragem de 1500 comprimidos do lote, sendo que o limite especificado para o quesito quebra, conforme a validação, é de no máximo 14 comprimidos, considerando o tamanho do lote, ou seja, o lote 2020X01 foi reprovado na inspeção.

Após a identificação dessa não conformidade, o setor da Garantia de Qualidade foi informado e o desvio foi formalmente registrado no sistema de gerenciamento de desvios de qualidade utilizado pela empresa e iniciou-se a investigação do problema.

O histórico de desvios envolvendo o produto $\mathrm{X}$ foi avaliado e pode ser visto na Figura 1, representado por meio de um Diagrama de Pareto, uma das ferramentas básicas da qualidade. Os da- dos deste histórico foram extraídos do sistema de gerenciamento de desvios utilizado pela empresa.

Figura 1. Diagrama de Pareto dos desvios de qualidade envolvendo o produto X entre 2019 e 2020

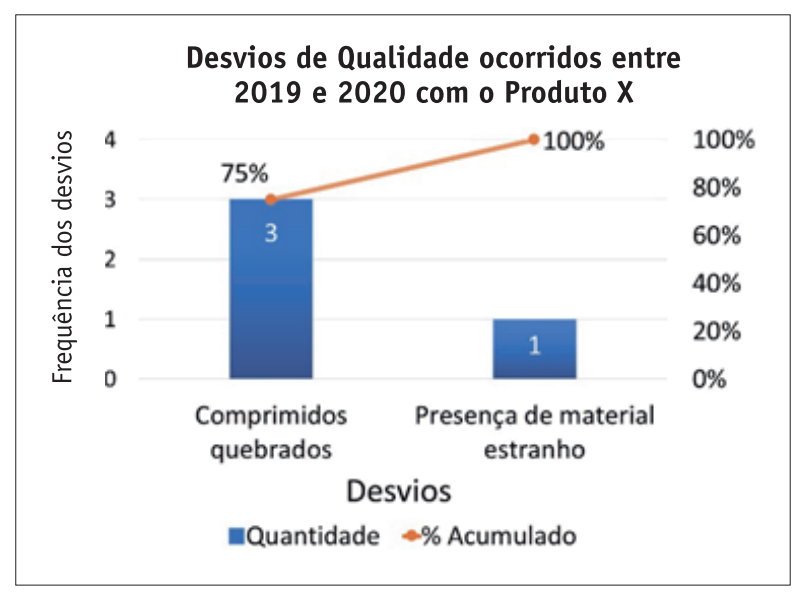

O Diagrama de Pareto é uma ferramenta que possibilita a visualização da frequência de determinadas falhas evidenciando a maior ocorrência. Esta ferramenta consiste em um gráfico que dispõe das frequências das ocorrências em ordem decrescente da esquerda para a direita, fornecendo uma visão de onde se deve tomar ações para melhoria do processo $(2,6)$. Entre 2019 e 2020 foi realizada a produção de 31 lotes do medicamento $\mathrm{X}$, e conforme pode ser visto na Figura 1, neste mesmo período (entre 2019 e 2020) o produto X apresentou 4 desvios no total, sendo um desvio relacionado à presença de material estranho e três com defeitos de quebra. Foi feita investigação para todos os desvios, e em relação ao material estranho encontrado, foi identificado pelo time de manutenção que este material se referia à uma peça do equipamento de produção que foi danificada durante o uso, e as medidas de correção e prevenção foram tomadas; trata-se de um caso isolado.

Por meio do Diagrama de Pareto fica evidente que a maior ocorrência é de comprimidos quebrados, representando $75 \%$ dos desvios, enquanto que a presença de material estranho equivale a $25 \%$ dos desvios. Dessa forma, fica claro que o defeito de comprimidos quebrados deve ser solucionado para redução considerável da reincidência dessa não conformidade. 
Os dados de produção dos lotes que apresentaram o defeito de quebra foram extraídos do sistema de gerenciamento de desvios e foram comparados com o lote em investigação (2020X01) para identificação de alguma similaridade que indicasse a causa da quebra.

O Diagrama de Ishikawa é utilizado para identificação das possíveis causas de um problema, separando-os por categorias (5). A construção começa com a identificação do efeito que se pretende investigar, realizando um brainstorm para determinar as possíveis causas de uma não conformidade e agrupando-as em seis categorias, a saber: Máquina, Mão de obra, Método, Meio ambiente, Medição e Material (7), conforme a Figura 2.

Sendo assim, a avaliação dos itens foi realizada com equipe de trabalho para identificação de causas que possam impactar na qualidade do produto. Cada item avaliado está descrito conforme a seguir.

Máquina. Conforme evidência apresentada pela equipe responsável, a calibração e manutenção preventiva dos equipamentos estavam dentro do prazo, e não houve abertura de pedidos de manutenção do equipamento durante o processo de fabricação do lote.

O operador e o técnico de punções apresentaram evidência de que o ferramental da compressora utilizado estava em boas condições e dentro do tempo de vida útil do equipamento.

Figura 2. Representação do Diagrama de Ishikawa aplicado ao fluxo de produção

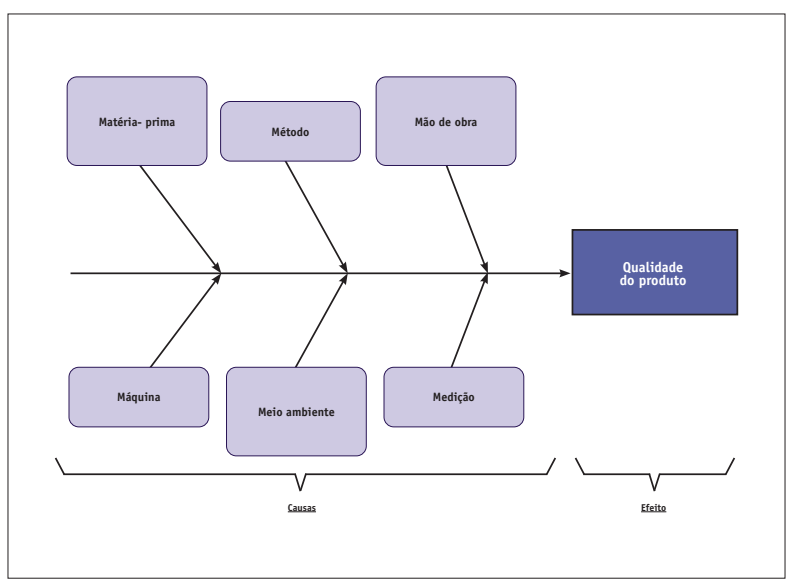

Material. Os fornecedores das matérias-primas utilizadas estavam qualificados. Todas as matérias-primas passam por um processo rígido pelo laboratório de Controle de Qualidade e todos os resultados das análises estavam dentro da especificação.

Foi verificado e todos os volumes das matérias-primas foram corretamente pesados e adicionados na fabricação do lote, ou seja, a quantidade utilizada estava de acordo com a formulação do medicamento.

Mão de Obra. Todos os operadores envolvidos nos processos estavam devidamente treinados nos procedimentos aplicáveis e não relatam dúvidas.

Meio Ambiente. Todas as salas de manufatura possuíam um sistema de controle de umidade, temperatura e pressão atmosférica do ambiente, sendo que um alarme sonoro e visual era ligado quando algum parâmetro ficava diferente do especificado. Foi verificado no sistema e todos os parâmetros permaneceram de acordo com o estabelecido.

Medição. Todos os equipamentos de análise de Controle em Processo (CEP) estavam calibrados e todos os processos estavam validados, garantindo a segurança dos resultados.

Método. Todos os processos possuíam um Procedimento Operacional Padrão (POP) que instruía como realizar a etapa conforme as Boas Práticas de Fabricação. Todos os POP estavam dentro do prazo de revisão.

Todas as etapas de produção possuíam instruções que foram seguidas conforme determinado. O processo estava validado e todos os CEP estavam conformes. A especificação de dureza era de 100 a $300 \mathrm{~N}$, entretanto foi identificado que os lotes que apresentaram dureza mais próxima do limite inferior, ainda que estivessem dentro do limite mínimo especificado, apresentaram maior facilidade para quebra.

Ao analisar os resultados de CEP, que são testes realizados durante a produção do produto para garantir o controle dos parâmetros, foi identificada similaridade dos resultados de dureza entre os lotes que apresentaram desvio de quebra, conforme Folha de Verificação (Tabela 1). 


\section{lonfarma}

Tabela 1. Folha de Verificação de resultados de dureza dos lotes do produto $\mathrm{X}$ que apresentaram quebra entre 2019 e 2020.

\begin{tabular}{|l|c|}
\hline $\begin{array}{c}\text { Lote com defeito de quebra de } \\
\text { comprimidos }\end{array}$ & $\begin{array}{c}\text { Dureza Média } \\
(\mathbf{1 0 0}-\mathbf{3 0 0} \mathbf{N})\end{array}$ \\
\hline $2019 \times 12$ & 156 \\
\hline $2019 \times 13$ & 134 \\
\hline $2019 \times 14$ & 128 \\
\hline $2019 \times 16$ & 146 \\
\hline $2020 \times 01^{*}$ & 149 \\
\hline
\end{tabular}

*Lote em investigação.

A Folha de Verificação é uma ferramenta em que diversos dados podem ser organizados de forma livre, conforme a necessidade do elaborador $(8,9)$. Por meio dos dados demonstrados pela Folha de Verificação (Tabela 1), é possível confirmar que o teste de dureza apresentou resultados dentro da especificação, que é de $100 \mathrm{~N}$ a $300 \mathrm{~N}$, conforme estudos validados; porém, a média de cada lote ficou mais próxima do limite inferior, sendo que a maior dureza média encontrada foi de 156 N. Devido à possibilidade de choques mecânicos durante o manuseio do produto nas diversas etapas de fabricação, embalagem, armazenamento, transporte e distribuição, a avaliação da resistência do comprimido ao esmagamento se torna essencial e é avaliada por meio do teste de dureza (10). O teste consiste em medir a força necessária para esmagar o comprimido, sendo Newtons (N) uma das unidades de medida (11).

Com a similaridade entre resultados de dureza dos lotes que apresentaram defeito de quebra, foi decidido realizar a comparação da dureza de todos os lotes produzidos entre 2019 e 2020; estes resultados foram obtidos a partir da documentação de cada lote. Para tal, o Gráfico de Controle foi elaborado (Figura 3).

O gráfico de controle é utilizado para mostrar tendências dos pontos de observação podendo colocar limites de tolerância para uma avaliação mais adequada. $\mathrm{O}$ gráfico permite a mensuração de quanto o processo pode estar ou não sob controle estatístico $(1,8)$.

Figura 3. Dureza média de cada lote do produto X produzido entre 2019 e 2020.

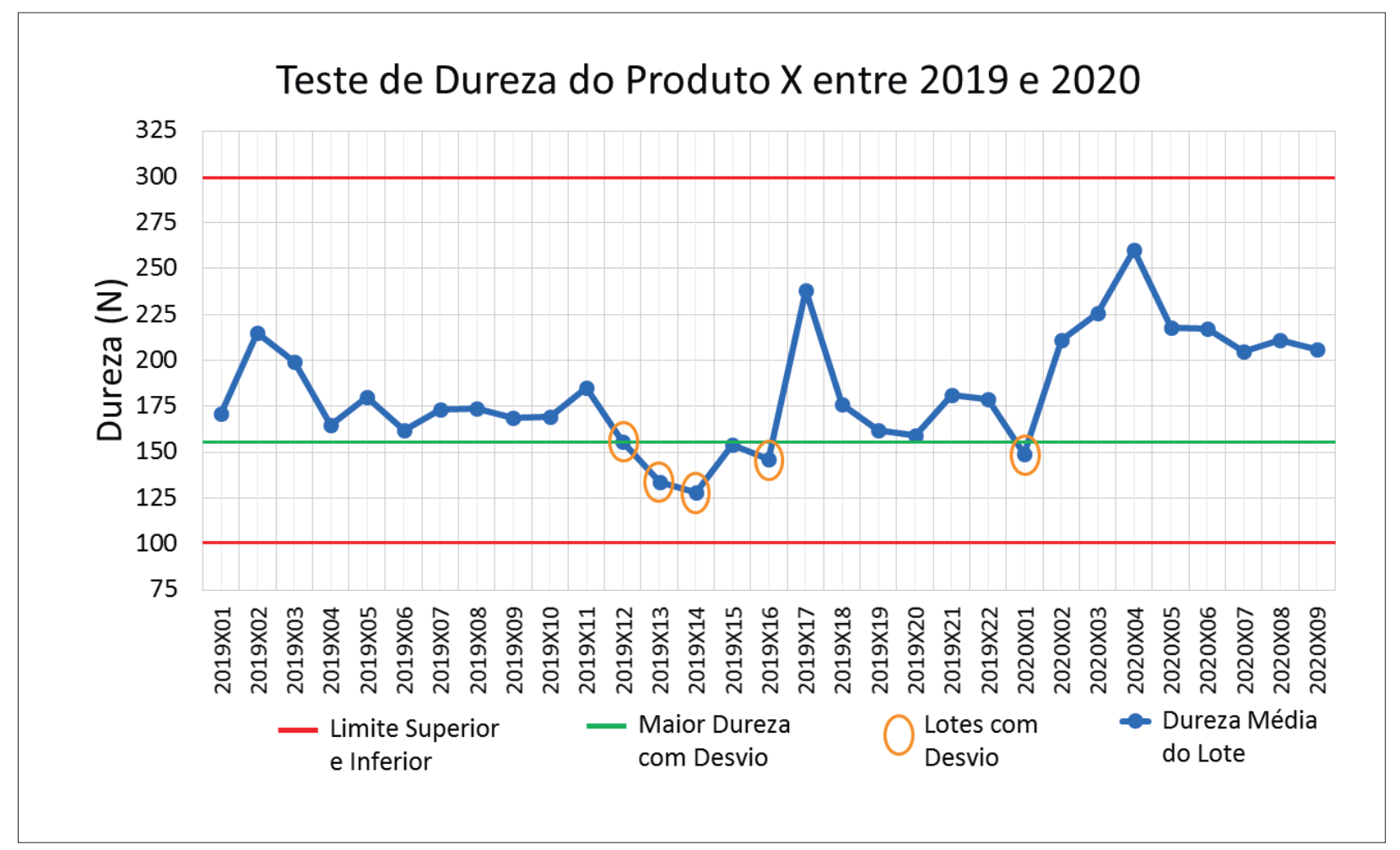


Na Figura 3 os lotes que apresentaram defeito de quebra estão sinalizados em laranja. Com este Gráfico de Controle, pode-se identificar que os lotes com defeito de quebra estão localizados na parte inferior do gráfico, ou seja, a dureza está mais baixa se comparada aos demais lotes que não apresentaram comprimidos quebrados. Sendo assim, fica evidente que os resultados baixos de dureza dos comprimidos podem facilitar a ocorrência de quebra dos mesmos, enquanto os lotes com dureza próximas ou superiores a $200 \mathrm{~N}$ obtiveram resultados satisfatórios.

Após análise dos dados, todos os itens avaliados no processo produtivo estavam conforme os requerimentos internos da empresa. Por mais que os resultados de dureza estivessem dentro da especificação do produto (100 a $300 \mathrm{~N})$, todos os lotes com defeito de quebra apresentaram a média de dureza abaixo de $156 \mathrm{~N}$, ou seja, apresentaram o resultado mais próximo do limite inferior. Como a dureza está diretamente relacionada com a resistência aos choques mecânicos, pode-se inferir que seu resultado baixo facilitou a quebra dos comprimidos.

Após a identificação da causa raiz, ações corretivas e preventivas foram discutidas para mitigação do problema.

Também conhecidas como CAPA, as ações corretivas e preventivas devem ser identificadas e implementadas em resposta às investigações de desvio de qualidade. Conforme a RDC no 301, de 21 de agosto de 2019, Ação Corretiva e Ação Preventiva (CAPA) trata-se de uma definição do plano de ação e sua implementação, com o objetivo de eliminar a causa raiz de eventos passados (como desvios, etc), evitando-se reincidências. Ações corretivas são medidas para tratar e eliminar a causa raiz do desvio já ocorrido, e as ações preventivas são medidas para evitar que um desvio volte a ocorrer (3).
Como ação corretiva, o lote $2020 \mathrm{X} 01$ foi encaminhado para revisão de $100 \%$ de todos os comprimidos. Este procedimento foi validado, os operadores envolvidos foram devidamente treinados e todos os comprimidos com defeitos foram removidos do lote, podendo então o lote ser encaminhado para o setor de embalagem.

Como ação preventiva, após reunião com a equipe de trabalho foi sugerido à empresa fabricar os próximos lotes buscando a dureza próxima ou superior a $200 \mathrm{~N}$ conforme histórico do produto, e acompanhamento para verificar como a dureza e a quebra dos comprimidos se comportam. Este acompanhamento deverá ser realizado pelos próximos seis meses, e ao término deste prazo um relatório deverá ser elaborado demonstrando os resultados e a conclusão, assim como as próximas ações a serem realizadas.

\section{CONCLUSÃO}

Cada vez mais as empresas precisam garantir que seus produtos tenham qualidade e segurança, e o setor da Garantia da Qualidade possui um importante papel neste objetivo.

Mesmo com controles durante os processos e uma equipe capacitada, os desvios de qualidade podem ocorrer, e para a investigação desse desvio diversas ferramentas de qualidade podem ser utilizadas para identificação da causa raiz.

As ferramentas de qualidade são essenciais para a investigação de um desvio de qualidade, pois são ferramentas que podem organizar os dados e distribuí-los de forma a facilitar a interpretação dos mesmos. Sendo assim, o treinamento da equipe responsável pelos desvios de qualidade se faz necessário para a aplicação correta das ferramentas da qualidade e consequentemente avaliação efetiva das não conformidades, resultando em produtos com maior qualidade, segurança e eficácia. 


\section{REFERÊNCIAS}

1. Ascenção TS. Aplicação das ferramentas da qualidade na avaliação de desvios na indústria farmacêutica. [Trabalho de Conclusão de Curso]. [Rio de Janeiro (RJ)]: Instituto de Tecnologia em Fármacos, Fundação Oswaldo Cruz; 2019.60p.

2. Palmeira AP, Rau C. Ferramentas de qualidade no gerenciamento de reclamações de mercado na Indústria Farmacêutica. Acta Ciência Saúde. 2014;3(2):109-131.

3. BRASIL. Resolução da Diretoria Colegiada RDC n 301 , de 21 de agosto de 2019. Dispõe sobre as Diretrizes Gerais de Boas Práticas de Fabricação de Medicamentos. Diário Oficial da União, Brasília (DF); 2019 Ago 22. Seção 1:64.

4. Boltic Z, Ruzic N, Jovanovic M, Petrovic S. Measuring the performance of quality assurance processes: pharmaceutical industry deviation management case study. Accredit Qual Assur. 2010;11(15):629-636. DOI: 10.1007/s00769-0100709-2

5. Silva JL. Aplicação das ferramentas da qualidade para melhoria de processos produtivos: estudo de caso em um centro automotivo. In: $37^{\circ}$ Encontro Nacional de Engenharia de Produção; 2017 out 10-13; Joinville, Santa Catarina (SC): Associação Brasileira de Engenharia de Produção (ABEPRO); 2017. p. 1-16.

6. Coelho FP, Silva AM, Maniçoba RF. Aplicação das ferramentas da qualidade: estudo de caso em pequena empresa de pintura. Rev Fatec Zona Sul. 2016;1(3):31-45.
7. Gomes AC, Ferreira AR, Silva EB. Produção de alimentos na indústria: principais ferramentas da qualidade. $\operatorname{In}: 27^{\circ}$ Encontro Nacional de Engenharia de Produção; 2017 out 10-13; Joinville, Santa Catarina (SC): Associação Brasileira de Engenharia de Produção (ABEPRO); 2017. p. 1-13.

8. Abrão OJ, Cardoso AA. Utilização do controle estatístico de processo atrelado às ferramentas da qualidade no monitoramento do peso das embalagens do produto final: um estudo de caso em uma indústria química. Braz. J. Develop. 2020;9(6):65241-65257. DOI: 10.34117/ bjdv6n9-091

9. Mariani CA. Método PDCAe ferramentas da qualidade no gerenciamento de processos industriais: um estudo de caso. INMR. 2007 2(2):110-126.

10. Peixoto MM, Santos-Júnior AF, Santos CA, Caetité-Júnior E. Avaliação da qualidade de comprimidos de captopril dispensados em Feira de Santana - BA. Infarma.1314(16):69-73.

11. BRASIL. Farmacopeia Brasileira, volume 01. $6^{\mathrm{a}}$ Ed. Brasília: Agência Nacional de Vigilância Sanitária. 2019. 